\title{
Gestão por competência dos profissionais através de um modelo multicritério construtivista
}

\author{
Felipe Tiago Eing Engelke Back \\ Universidade Federal de Santa Catarina - UFSC \\ febacks@gmail.com

\section{Leonardo Ensslin} \\ Universidade Federal de Santa Catarina - UFSC \\ ensslin@deps.ufsc.br \\ Sandra Rolim Ensslin \\ Universidade Federal de Santa Catarina - UFSC \\ sensslin@deps.ufsc.br
}

\section{RESUMO}

O presente trabalho visa obter um Modelo de Avaliação de Desempenho que serve como ferramenta de apoio ao Gestor durante a alocação de recursos tendo em consideração o grau de conhecimento, habilidade e aptidão das pessoas quando do desenvolvimento de seus projetos, e para isso utiliza como instrumento de intervenção, o método Multicritério de Apoio à Decisão Construtivista (MCDA-C). Trata-se de um estudo de caso aplicado em uma empresa Multinacional de Eletrodomésticos que ao final, permitiu identificar: as três grandes áreas de preocupação do gestor para quem o modelo foi construído; 76 critérios/KPIs que representam as preocupações associadas aos valores do Gestor; as escalas ordinais e cardinais para cada critério com seus níveis de referência, e realizar a integração através das taxas de compensação; realizar e avaliar o diagnóstico da situação atual da alternativa analisada com 32 em escala cardinal; e por fim apresentar o modelo com proposta de ações de aperfeiçoamento que elevaram sua avaliação em para 82.

Palavras-chave: Avaliação de desempenho, Gestão de Recursos Humanos, Análise de decisão multicritério, MCDA-C.

\begin{abstract}
This paper aims to construct a Performance Evaluation model that serves as a tool to support the Manager during the resource allocation taking into account the knowledge degree, skills and abilities for project development, and as an instrument of intervention the Multicriteria Decision Aid - Constructivist method (MCDA-C). It is case study applied on the global Home Appliances company that in the end, has identified: three major dimensions of concern to the manager for whom the model was constructed; 76 criteria/KPIs that represent the concerns associated with the Manager values; constructing cardinal and ordinal scales for each criterion with its reference levels and achieve the integration through the compensation rates; implement and evaluate the diagnosis of the current situation with of the alternative analyzed with 32 points on a cardinal scale; and present the model with proposed actions to improve its assessment that raised to 82 .
\end{abstract}

Keyword: Performance evaluation, Human Resource Management, Multicriteria decision analysis, MCDA-C.

\section{Introdução}

Os consumidores atuais esperam por produtos com propriedades singulares as suas necessidades além dos critérios tradicionais, preço, qualidade, inovação, agilidade, meio ambiente e responsabilidade social, durante o processo de seleção e aquisição de bens. Todo esse cenário tem transformado o mercado e gerando novos desafios aos fabricantes, além da própria competição acirrada pela globalização, compartilhamento de informações em tempo real e a crescente valorização das inovações. Nesse cenário, toda a cadeia necessita ser repensada no sentido de alavancar novas estratégias visando o atendimento dessa nova demanda com o alcance de um diferencial competitivo (CAI et al., 2009; CHAN, 2003; JIMENEZ; LORENTE, 2001). 
Com relação às novas exigências dos consumidores, destaca-se à importância no atendimento das demandas individuais, resultando num mercado crescente e determinado em adquirir produtos personalizados aos seus desejos. Em contrapartida para os fabricantes, aumenta a complexidade e consequentemente o número de projetos com o objetivo de cada vez mais, atender seus consumidores de forma individual. É claro que os desafios quanto à redução de desperdícios, recursos e tempo de lançamento de projetos e entre outros fatores de gestão, são considerados básicos no processo. Desse modo, os mecanismos de gestão que eram considerados consolidados desde outrora, tiveram que ser repensados uma vez que o problema se modificou (RABECHINI; CARVALHO; LAURINDO, 2002).

Este artigo está inserido no contexto de alocação de recursos tendo em consideração o grau de conhecimento, habilidade e aptidão das pessoas quando do desenvolvimento de seus projetos, buscando-se diferenciação competitiva face aos tradicionais métodos baseados nos cargos e tempo de empresa. Assim, emerge a pergunta de pesquisa: Como realizar a alocação de pessoas para atender as demandas de projetos para o contexto avaliado com visão sistêmica e alavancar vantagem competitiva para organização?

A fim de responder a pergunta de pesquisa, apresenta-se como objetivo a realização da gestão por competência dos profissionais através de um modelo multicritério construtivista para atender as demandas de conhecimento dos projetos e criar assim um diferencial competitivo. Tendo em vista atender ao objetivo proposto, seguiram-se as fases do método MCDA-C, Estruturação, Avaliação e Recomendações, destacando-se a contextualização do problema, definição dos atores envolvidos, identificação dos critérios/KPIs segundo o juízo de valores do decisor no contexto analisado, criação das escalas na forma ordinal e posteriormente a transformação em escala cardinal, mensuração com diagnóstico numérico e gráfico da situação atual (status quo) tanto em nível local quanto global e por fim, realizar ações de aperfeiçoamento tendo o pleno entendimento do impacto de cada decisão tomada.

O presente artigo tem como área de aplicação a Gestão de Recursos Humanos através do método MCDA-C, por isso faz-se uma breve apresentação de ambos no Arcabouço Teórico antes de apresentar o estudo de caso propriamente dito que detalhará todas as etapas do método selecionado com os resultados alcançados.

\section{Referencial Teórico}

O arcabouço teórico é dividido em duas partes: Inicialmente com conceito e algumas características da Gestão de Recursos Humanos e no segundo momento, aborda o instrumento de intervenção utilizado neste trabalho, o método MCDA-C.

\subsection{Gestão da Alocação de Recursos Humanos}

A Gestão dos Recursos Humanos é uma filosofia de apoio a decisão que contribui diretamente no alcance dos objetivos estratégicos e também é amplamente encontrada na literatura internacional como Human Resource Management "HRM" (BAIRD; MESHOULAM, 1988; JACKSON; SCHULER, 1999). A Gestão de Recursos Humanos é caracterizada pelo seu foco no entendimento do comportamento das pessoas face à um determinado resultado esperado (BUCK; WATSON, 2002). Já os pesquisadores Beardwell e Claydon (2007), enfatizam a questão estratégica quando da utilização das pessoas com base em suas habilidades e como interagem com os objetivos organizacionais.

Segundo Wright et al. (2001), é fundamental que estejam alinhadas as ações individuais com o desenvolvimento dos recursos críticos para otimizarem os ganhos para as organizações. Com relação a complexidade no processo de alocação de pessoas, Hendriks, Voeten e Kroep (1999), destacam a presença de atividades com múltiplos critérios e heterogêneas, dos quais os objetivos não estão claros. No sentido de alcançar vantagem competitiva, as iniciativas de construção de uma estratégia personalizada para alocação de pessoas, serão dificilmente imitadas por outras organizações (LADO; WILSON, 1994).

Com relação as novas exigências dos consumidores, destaca-se à importância no atendimento das demandas individuais, pois há um mercado crescente e decido por adquirir produtos personalizados aos seus desejos. Além disso, outros fatores-chave como a globalização, mercados emergentes como China, Índia, América Latina, Europa Oriental e outros. Toda essa tendência contribui para o aumento dos estudos referentes à Gestão da Alocação de Recursos Humanos (BRESTER et al., 1996). Há vários estudos científicos publicados baseados no impacto da Gestão da Alocação dos Recursos Humanos no desempenho competitivo das empresas (HUSELID, 1995).

Com relação à alocação de pessoas para atender a demanda de projetos supracitada, os gestores são desafiados a tomarem decisões em ambientes em que muitas vezes não tem clareza dos objetivos, não conseguem desenvolver mecanismos para entender o problema em questão e também o impacto de cada decisão no resultado global (GANN; SALTER, 2000).

Além disso, os processos e ferramentas tradicionais são amplamente difundidos no ambiente de gestão. Há 
uma forte tendência pela utilização dos modelos que teoricamente podem oferecer uma solução ótima e são imitados pelas grandes empresas. Contudo, restringemse às oportunidades inovadoras do gestor, alcançando resultados em que o decisor tem conhecimento de cada decisão e sabe o que fazer para buscar aperfeiçoamentos, a fim de obter diferenciação competitiva.

Com o objetivo de responder a pergunta de pesquisa e inserido no contexto da Gestão de Recursos Humanos, adotou-se o método Multicritério de Apoio à Decisão Construtivista (MCDA-C) por ter em seu âmago, expandir o conhecimento do decisor em ambientes singulares, complexos e conflituosos (ENSSLIN et al., 2010). Os pesquisadores de destaque e amplamente citados na literatura como Skinner (1986), Keeney (1992), Roy (1993; 1996; 2005), Landry (1995), Bana e Costa et al. (1999), Zimmermann (2000), Shenhar (2001), Steward (2005), Igarashi et al. (2008), adotaram as mesmas premissas citadas acima no processo de construção dos seus modelos.

\subsection{O Método MCDA-C}

A consolidação do método MCDA-C (Metodologia Multicritério de Apoio à Decisão - Construtivista) surge como destaque no mundo científico principalmente através das recentes publicações (últimos 20 anos) de Keeney (1992), Bana e Costa (1993; 1999), Landry (1995), Roy (1996), Ensslin et al. (2000) e Ensslin et al. (2010) como instrumento de gestão, embora exista a 200 anos.

Adicionalmente, o método MCDA-C apresenta diferença face aos tradicionais MCDAs, principalmente pela fase de Estruturação, na qual o problema é contextualizado, há identificação do sistema de atores, foco na participação e expansão do conhecimento do tomador de decisão (decisor) sobre o contexto. No caso das tradicionais MCDAs, restringem-se às fases de Formulação e Avaliação durante o apoio à decisão, tendo um agrupamento de aspectos definidos como objetivos (decisor com pouca ou nenhuma participação), visando selecionar a alternativa considerada ótima face às alternativas previamente estabelecidas (KEENEY, 1992; ROY; BOUYSSOU,1993; ROY, 1996; GOODWIN; WRIGHT, 1998).

No caso do método MCDA-C que é uma ramificação da MCDA-C tradicional, exatamente por considerar a fase de Estruturação e auxiliar o decisor com uma ferramenta de apoio a decisão em ambientes considerados conflituosos, incertos e complexos.

Roy (1994; 1996; 2005), caracteriza os pesquisadores de acordo com a utilização do MCDA quanto à lógica de investigação em 3 grandes grupos: racionalistas, axiomáticos ou prescritivistas e construtivistas.

O MCDA-C é considerado diferenciado por ter abordagem construtivista que privilegia o processo visa colocar a ciência a serviço da expansão do conhecimento do tomador de decisão e assim, suportá-lo no apoio a decisão, permitindo o entendimento pleno sobre o impacto de cada decisão no resultado final. Com o intuito de atender aos objetivos, o MCDA-C é desenvolvido em três fases seqüenciais, mas interativas: Estruturação, Avaliação e Recomendações, conforme ilustrado na Figura 1. 


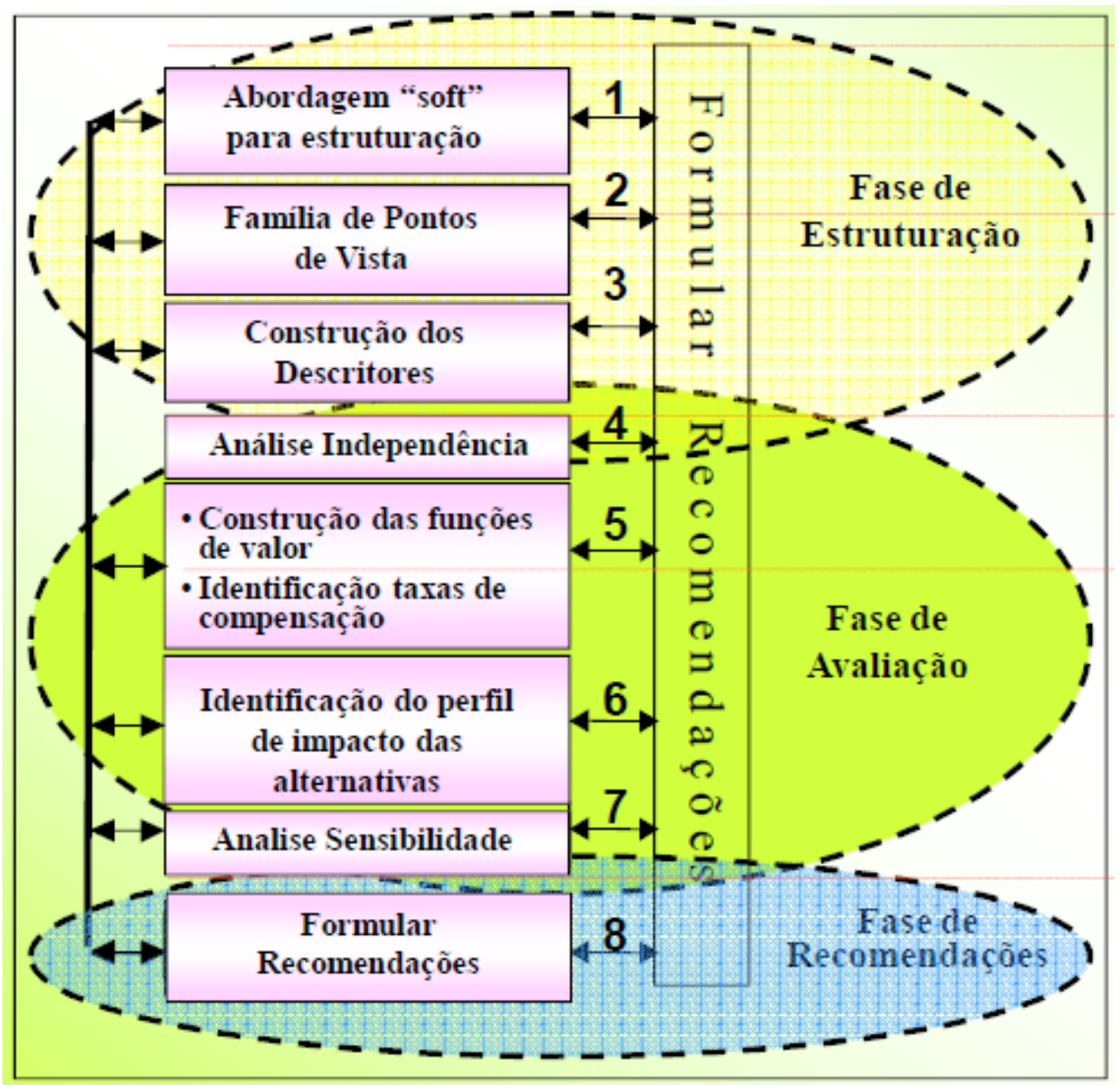

Figura 1 - Fases do MCDA-C

Fonte: Ensslin, Montibeller e Noronha (2001).

Para o método MCDA-C não existe solução ótima, ou seja, há sempre espaço para aperfeiçoamentos. Nesse contexto, o método é recursivo e é permitida a elaboração de recomendações em todas as etapas do processo.

\section{Enquadramento Metodológico}

Esta pesquisa é caracterizada no que tange a natureza do artigo como aplicada ou tecnológica uma vez que o estudo tem foco no apoio à Gestão por competência dos profissionais através de um modelo multicritério construtivista quando do desenvolvimento de projetos. Quanto ao enquadramento do objetivo da pesquisa e a natureza dos objetivos é definida como pesquisa exploratória, na qual Gil (1999) caracteriza pelo esforço em criar-se maior familiaridade com o problema e torná-lo mais explícito.

Referente ao processo de pesquisa no que tange à abordagem do problema, observaram-se características qualitativas e quantitativas. A qualitativa por permitir a expansão do conhecimento do contexto, através da identificação dos critérios e a construção de escalas ordinais. Já a quantitativa, tendo em consideração o uso de modelos matemáticos para transformar as escalas ordinais em cardinais e identificação das taxas de compensação. As taxas de compensação definem o grau de atratividade entre os critérios identificados segundo o julgamento de valores do decisor. Essas taxas servem para integrar (agregar) os critérios do modelo e permitir uma avaliação de desempenho global ou nível estratégico (ENSSLIN; VIANNA, 2008).

No processo de pesquisa, o procedimento para coleta de dados é de origem primária uma vez que as informações foram adquiridas através das entrevistas nas reuniões formais e informais, principalmente com o Gestor (decisor). O decisor tem papel fundamental no processo, pois ele tem limite de conhecimento quanto ao problema analisado, mas concentra o conhecimento inicial com relação aos aspectos que devem ser considerados como relevantes ao estudo. 
O artigo é caracterizado como estudo de caso, pois foi aplicado numa empresa Multinacional de Eletrodomésticos segundo o julgamento de valores do seu Líder de Tecnologia. Por fim, utiliza-se como instrumento de pesquisa para apoiar o gestor na alocação de pessoas, o método MCDA-C. Além disso, destaca-se o uso do método MacBeth para identificar a diferença de atratividade entre os níveis da escala e o programa M-MacBeth.

\section{Fase de Estruturação: Gestão por competência dos profissionais através de um modelo multicritério construtivista}

Em abordagens como a do presente artigo que contempla diversas variáveis, lida com situações complexas, influência de interesses dos atores envolvidos e que esses fatores impactam isoladamente ou em conjunto no resultado final, o método MCDA-C é recomendado. O método é composto por oito etapas que podem ser visualizadas através da Figura 1 e serão explicitadas ao longo do artigo.

A Fase de Estruturação é a etapa macro do método MCDA-C que é fundamental para o processo (base) e diferencial frente às demais MCDA, sendo composta por: abordagem soft para estruturação, família de pontos de vista e construção dos descritores.

\subsection{Abordagem Soft para Estruturação}

A abordagem soft para estruturação é a primeira etapa do método MCDA-C e nela tem-se por objetivo contextualizar o problema, identificar os atores envolvidos, estabelecer o rótulo e sumário que seja composto por: problema; importância; objetivo; instrumento de intervenção; e resultados esperados.

Com relação à contextualização, o estudo de caso foi desenvolvido numa empresa Multinacional que desenvolve e fabrica eletrodomésticos cuja filial envolvida é sediada no Sul do Brasil. A empresa em questão é focada no consumidor e esse por sua vez, está cada vez mais exigente quanto ao atendimento as suas singularidades, custos envolvidos, qualidade e outros vários fatores. Com isso, o portfólio de projetos cresce com o intuito de atender a essa demanda, além do desafio agregado de se reduzirem desperdícios, recursos e tempo de lançamento de produtos. A empresa possui 800 funcionários de um total de 7000 com escopo de trabalho relacionado ao desenvolvimento de projetos e todo esse recurso humano necessita de gestão.

Nesse contexto em que a demanda e a complexidade de projetos aumentam, ao passo que a disponibilidade de recursos se mantém e muitas vezes ainda há pressão por reduções, verifica-se a importância do uso eficiente dos recursos. Os sistemas de gestão tradicionais com base no cargo e não na competência acabam comprometendo esse balanço entre alocação e eficiência, além de não permitirem a visualização do impacto das decisões no resultado final por parte gestor quando da alocação dos recursos humanos. Emerge assim, a necessidade da construção de um modelo que leve em conta o grau de conhecimento dos profissionais e que sirva como ferramenta de apoio a gestão do decisor no momento da alocação de recursos humanos, permitindo-se ainda visualizar as conseqüências de suas decisões no contexto avaliado.

Tendo contextualizado o problema, seguiu-se para a identificação do sistema de atores conforme Quadro 1.

\begin{tabular}{|c|c|l|}
\hline \multirow{4}{*}{ Stakeholders } & \multicolumn{1}{|c|}{ Atores } & \multicolumn{1}{c|}{ Descrição dos atores } \\
\cline { 2 - 3 } & Decisor & Líder de Tecnologia \\
\cline { 2 - 3 } & Facilitador & Engenheiro de Produto \\
\cline { 3 - 4 } & \multirow{2}{*}{ Intervenientes } & Envolvidos com o projeto \\
\cline { 3 - 3 } & & Gerente de Tecnologia \\
\cline { 2 - 3 } & Líder de Subsistemas \\
\hline \multicolumn{2}{|c|}{ Agidos } & Colegas de trabalho e Funcionários da empresa \\
\hline \multicolumn{2}{|c|}{ Quadro 1 - Atores }
\end{tabular}

Fonte: Elaborado pelos autores (2010)

Com base no conhecimento até então construído, o facilitador e o decisor estabeleceram o rótulo "Gestão por competência dos profissionais através de um modelo multicritério construtivista" para o problema analisado tendo como intuito explicitar as preocupações do Líder de Tecnologia em uma forma resumida. 


\subsection{Família de Pontos de Vista}

A etapa de Família de Pontos de Vista inicia com a consolidação dos Elementos Primários de Avaliação (EPAs) que representam aqueles aspectos iniciais, desejos, objetivos e restrições face ao problema avaliado, e que foram extraídos do decisor.

Nesse momento é importante não restringir o decisor (similar ao raciocínio do Brainstorming), registrandose o maior número possível de EPAs. Foram realizadas reuniões formais com o decisor e outras conversas informais com os atores envolvidos. Desses encontros, foram identificados 80 EPAs.

Finalizado o processo acima, segue-se para a etapa seguinte do método MCDA-C que busca a expansão do conhecimento gerado pelos EPAs, transformando-os em conceitos. Um conceito necessita apresentar a direção preferencial pólo psicológico oposto do decisor (EDEN et al., 1992).

Assim, foram apresentados ao decisor os 80 EPAs gerador e para cada um, foi solicitado a ele que abordasse o tema no contexto que envolve os EPAs, conduzindo-se esta etapa através de questionamentos tais como: Qual o status atual? O que considera o melhor e também o pior desempenho? O que considera bom e ruim? Qual a intensidade de cada desempenho (o verbo citado no discurso tende a refletir a intensidade do conceito).

Finalizado o processo supracitado, foi possível estabelecer os 80 conceitos dos quais são ilustrados quatro no Quadro 2. Ressalta-se que a reticência [...] deve ser interpretada como "ao invés de", ou seja, o pólo presente tendo como exemplo o conceito 80 "Conhecer as entregas da etapa de decisão do negócio" é preferível ao seu corresponde oposto psicológico "Comprometer o cronograma do projeto por falha no planejamento".

\begin{tabular}{|c|l|}
\hline Conceito & \multicolumn{1}{c|}{ Descrição } \\
\hline C77 & $\begin{array}{l}\text { Fazer uso detalhado das ferramentas de En- } \\
\text { genharia... Comprometer as entregas do pro- } \\
\text { jeto ou condução das atividades }\end{array}$ \\
\hline C78 & $\begin{array}{l}\text { Garantir que seja liberado o projeto com qua- } \\
\text { lidade assegurada... Liberar o projeto sem } \\
\text { finalizar as análises necessárias }\end{array}$ \\
\hline C79 & $\begin{array}{l}\text { Fazer a entrega da etapa de decisão do negó- } \\
\text { cio... Conduzir o projeto em etapas posterio- } \\
\text { res sem finalizar as anteriores }\end{array}$ \\
\hline C80 & $\begin{array}{l}\text { Conhecer as entregas da etapa de decisão do } \\
\text { negócio... Comprometer o cronograma do } \\
\text { projeto por falha no planejamento }\end{array}$ \\
\hline
\end{tabular}

Quadro 2 - Conceitos

Fonte: Elaborado pelos autores.
O facilitador questiona o decisor em forma $U p$-Down quanto as suas grandes preocupações para o problema analisado de forma macro e definem algumas Áreas de Preocupações. Todos os conceitos criados são agrupados de acordo com seu conteúdo nessas áreas pré-estabelecidas.

\subsection{Construção dos Descritores}

Com a construção dos Descritores, é possível construir as escalas ordinais que servirão para avaliação do desempenho de uma alternativa selecionada, considerando-se as propriedades do contexto que operacionalizam os objetivos estratégicos.

\subsubsection{Mapas Cognitivos e Árvore de Pontos de Vista Fundamentais}

Na etapa Macro de Construção dos Descritores, tem-se a criação dos mapas cognitivos para que no primeiro momento seja estabelecida a Árvore de Pontos de Vista Fundamentais. Com relação aos mapas cognitivos (mapas de relação meios-fins) foi utilizado como ferramenta de apoio uma vez que permite a construção e expansão do conhecimento, durante o processo de identificar as relações de hierarquia e influência entre os conceitos (BANA E COSTA; ENSSLIN, 1999); (ENSSLIN et al., 2000); (ENSSLIN et al., 2010). É um processo que busca aprofundar o entendimento de cada conceito identificado com o objetivo de extrair do decisor o máximo de informações possível. Para essa coleta de informações, são sugeridas algumas perguntas-chave como: "Como obter o conceito?" e "Qual a importância do conceito?” (ENSSLIN et al., 2010).

Os questionamentos acima citados foram repetidos até que os atores envolvidos considerassem que todas as relações possíveis de causa e efeito tivessem sido atendidas. Cada conceito inicial foi rotulado de 1 a 499 e com os conceitos novos que emergiam durante o processo, com a identificação a partir de 500. O método MCDA-C considera importante a expansão do conhecimento e deste modo, ao incentivar a discussão com os atores sobre a problemática em questão e os conceitos iniciais, é comum o surgimento de novos conceitos.

O processo utilizado pelo MCDA-C é inicialmente divergente para evitar qualquer restrição à construção do conhecimento. Após estabelecidos os Mapas Cognitivos, utilizam-se Clusters para alcançar a convergência entre os conceitos, além de facilitar o entendimento posterior pois cada Cluster contém um agrupamento de preocupações do decisor. Por exemplo, na Figura 2, ao percorrer os ramos (linhas que ligam um conceito ao outro) desde o conceito inicial até os finais, foram identificados três Clusters: Ferramentas Finais, Testes Finais e Decisão do Negócio. No caso do Cluster “Decisão do 
Negócio", os conceitos 79 e 80 são conceitos base do conceito 547 que por sua vez está ligado ao conceito 544, desse modo puderem ser agrupados de forma a formar o Cluster "Decisão do Negócio".

De posse dos Clusters criados e todo o conhecimento construído com o processo supracitado, passou-se a etapa seguinte proposta pelo método MCDA-C que é a construção da Estrutura Hierárquica de Valor. Nessa etapa, busca-se extrair todas as informações dos Mapas Cognitivos para que de forma gráfica e sucinta, apresente os aspectos julgados como relevantes ao problema segundo o julgamento de valores do decisor. Nesse processo, oportuniza-se a expansão do conhecimento e a organização da informação de forma lógica (KEENEY, 1992).

Vale destacar que os Clusters devem ser: homogêneos; conciso; controlável; essencial; isolável; mensurável; não-redundante; e operacional (KEENEY, 1992); (ENSSLIN et al., 2001); (ROY, 2005); (ENSSLIN et al., 2010). Recomenda-se o desmembramento de cada Clusters até o atendimento das propriedades acima citadas para que os Clusters possam ser considerados na Estrutura Hierárquica de Valor que então recebe o nome de Ponto de Vista Fundamental - PVF.

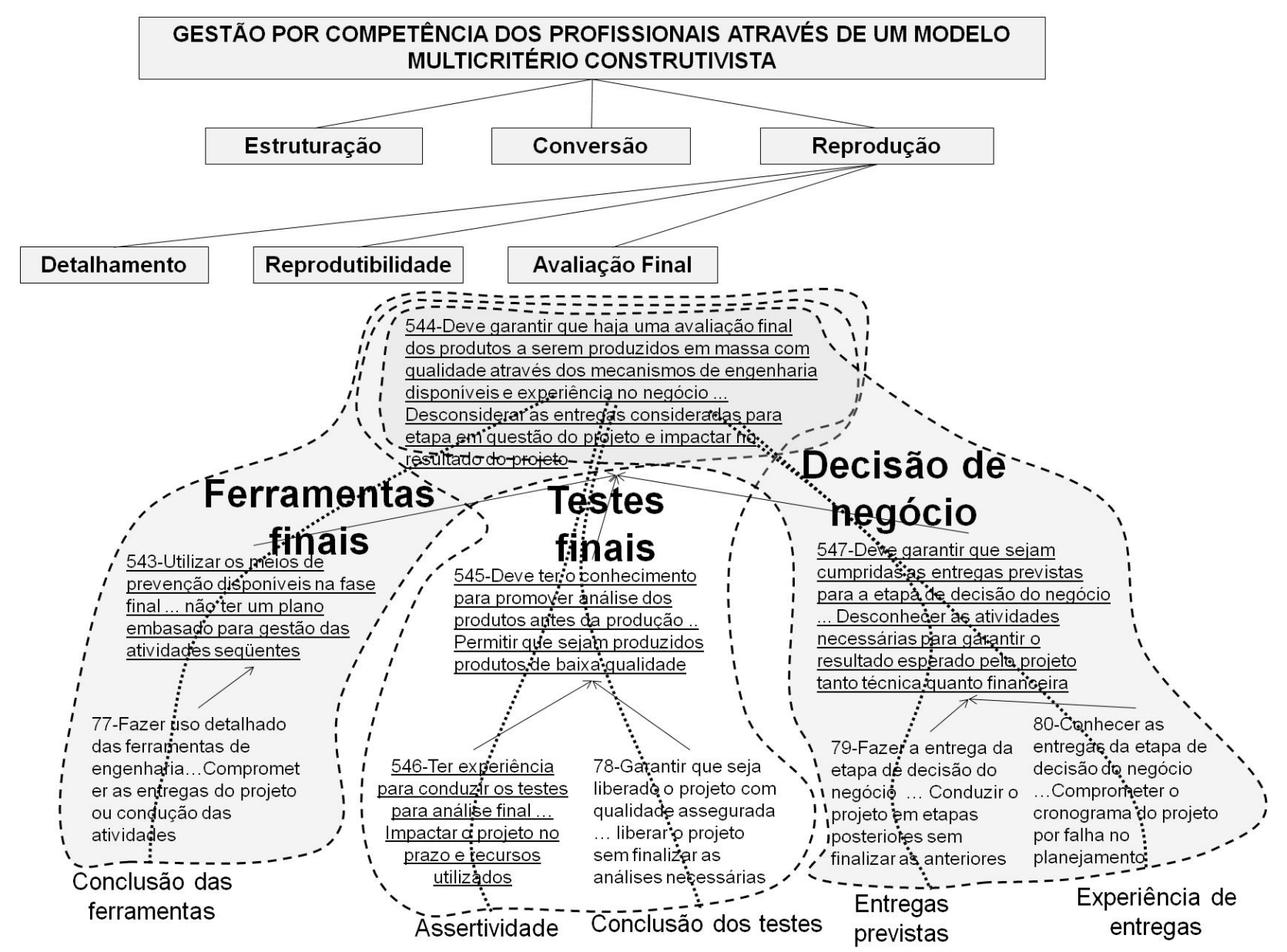

Figura 2 - Relação de Meios-Fins para os Clusters Ferramentas finais, Testes finais e Decisão de negócio

Fonte: O autor (2012)

\subsubsection{Estrutura Hierárquica de Valor e Descritores}

Estabelecidos os Pontos de Vista Fundamentais, temse por objetivo o estabelecimento dos Pontos de Vista Elementares (PVE). Para isso, utilizam-se os Mapas Cognitivos criados e adota-se o mesmo processo desenvolvido para os Clusters, porém dessa vez no nível dos
Clusters identificados. Ao agrupamento dessas preocupações dentro dos Clusters, são nomeadas como Subclusters e na Estrutura Hierárquica de Valor, recebem a denominação de Ponto de Vista Elementar (PVE). Os PVFs devem ser decompostos até que os PVEs possam ser mensurados de forma homogênea e não ambígua, representando o contexto analisado. 
No presente estudo, foram identificados nove Pontos de Vista Fundamentais dos quais foram obtidos através do processo supracitado. Finalizado esse processo, a próxima etapa é a Construção dos Descritores que são escalas ordinais geradas através da Estrutura Hierárquica de Valor e considerando todo o conhecimento adquirido, tendo como intuir realizar a mensuração posterior dos critérios/KPIs identificados. Utiliza-se um processo interativo com o Decisor para construir as escalas ordinais que representem todas as preocupações associadas aos valores do decisor para cada extremo inferior dos Subclusters.

Além disso, são estabelecidos os níveis de referência (Âncora). Bana e Costa et al. (1999), Ensslin, Dutra e Ensslin (2000), Ensslin et al. (2010) e Roy (2005) classificam esses níveis de referência como Nível Bom e Nível Neutro. Essas são as fronteiras do desempenho que são denominadas segundo suas performances: Excelência, Competitivo e Comprometedor. Assim, o Nível Bom serve como limitante entre as performances de Excelência e Competitiva. No caso do Nível Neutro, é a fronteira que separa a performance competitiva da considerada comprometedora.

\section{Fase de Avaliação}

Através da fase de Estruturação, anteriormente apresentada, foi possível construir um modelo que permitisse a realização de análises qualitativas para o contexto avaliado e que refletisse os aspectos considerados necessários e suficientes pelo decisor. Nesse modelo, foram criadas escalas ordinais (Descritores) através de símbolos numéricos para sua representação. Contudo, de acordo com Ensslin et al. (2001), Barzilai (2001) e Azevedo (2001), os números utilizados são somente símbolos alfa-numérico que não pertencem ao conjunto dos números reais. Além disso, ressalta-se que por se tratarem de símbolos alfa-numérico, os símbolos não podem ser utilizados para funções com operações matemáticas ou estatísticas.

Nesse contexto e com a finalização da fase de Estruturação do método MCDA-C, verifica-se a importância da fase seguinte que é a de Avaliação, sendo composta pelas etapas: Análise de Independência, Construção das Funções de Valor, Identificação das Taxas de Compensação, Identificação do Perfil de Impacto das Alternativas e Análise de Sensibilidade conforme Figura 1.

\subsection{Análise de Independência}

A expansão do conhecimento no decisor, posteriormente à identificação das escalas ordinais, é verificada através do processo de transformação das escalas ordinais em cardinais e dessa forma, permitirá a integração de todas as escalas do modelo.
Para realizar o processo supracitado, são utilizados no método MCDA-C, modelos compensatórios com o objetivo de integrar as escalas e assim permitir uma avaliação global. Segundo Roy (1993), as Abordagens de Critério Único de Síntese são características dos modelos gerados pelo MCDA-C.

Entretanto, antes de realizar o processo de transformação supracitado e passar a etapa seguinte (Função de Valor), deve-se verificar a isolabilidade (independência preferencial) de todas as escalas (Descritores), pois o os modelos do método MCDA-C só admitem taxas de compensação constantes e isso demanda que os critérios sejam preferencialmente independentes. Para tanto é aplicada a Independência Preferencial Cardinal - IPC às escalas no intervalo construído (ENSSLIN et al., 2001).

No presente estudo, foi aplicado o teste de isolabilidade para todos os Descritores, o que garantiu a Independência Preferencial Cardinal (Isolabilidade) para todas as escalas e assim o atendimento aos requisitos do método MCDA-C.

\subsection{Funções de Valor}

Esta etapa começa com a definição do método para realizar a transformação da escala ordinal em Função de Valor (escala cardinal). Para o MCDA-C, as escalas cardinais devem ser construídas de forma a garantir que a diferença de atratividade entre os níveis da escala esteja de acordo com o grau de intensidade julgado pelo decisor. Tal necessidade pode ser atendida com a utilização de métodos como: Bisseção, Pontuação Direta e MacBeth (ENSSLIN et al., 2001). Para o presente estudo de caso, tendo em consideração a vasta aplicação dos pesquisadores no mundo científico e fundamentação teórica, será utilizado o método Macbeth com o auxílio do programa M-MacBeth.

Com relação ao Macbeth e com o objetivo de construir as funções de valor, é necessário que se crie uma matriz de julgamento semântica. Nessa matriz, questiona-se o decisor sobre suas preferências e grau de intensidade ao se comparar pares de descritores. O programa M-MacBeth oferece uma escala ordinal com 7 níveis de atratividade: nula, muito fraca, fraca, moderada, forte, muito forte e extrema. Após alimentar a matriz com o grau de atratividade para cada combinação, o programa gera automaticamente o campo de soluções que atenda ao julgamento de valores do decisor e desse conjunto, propõe uma com base em modelos de programação linear (BANA E COSTA et al., 2005). A escala gerada pelo programa deve ser legitimada pelo decisor e ainda ajustada, para passar de candidata a função de valor para o descritor analisado. 
Além disso, as Funções de Valor são normalizadas para os níveis de referência (Nível de Referência Neutro = "0" e Nível de Referência Bom = "100") a fim de torná-las comparáveis e permitir a construção do modelo global.
Para o presente estudo é demonstrada a transformação do descritor (escala ordinal) de um PVE - "Experiência em entregas" para obtenção de sua respectiva função de valor conforme Figura 3.

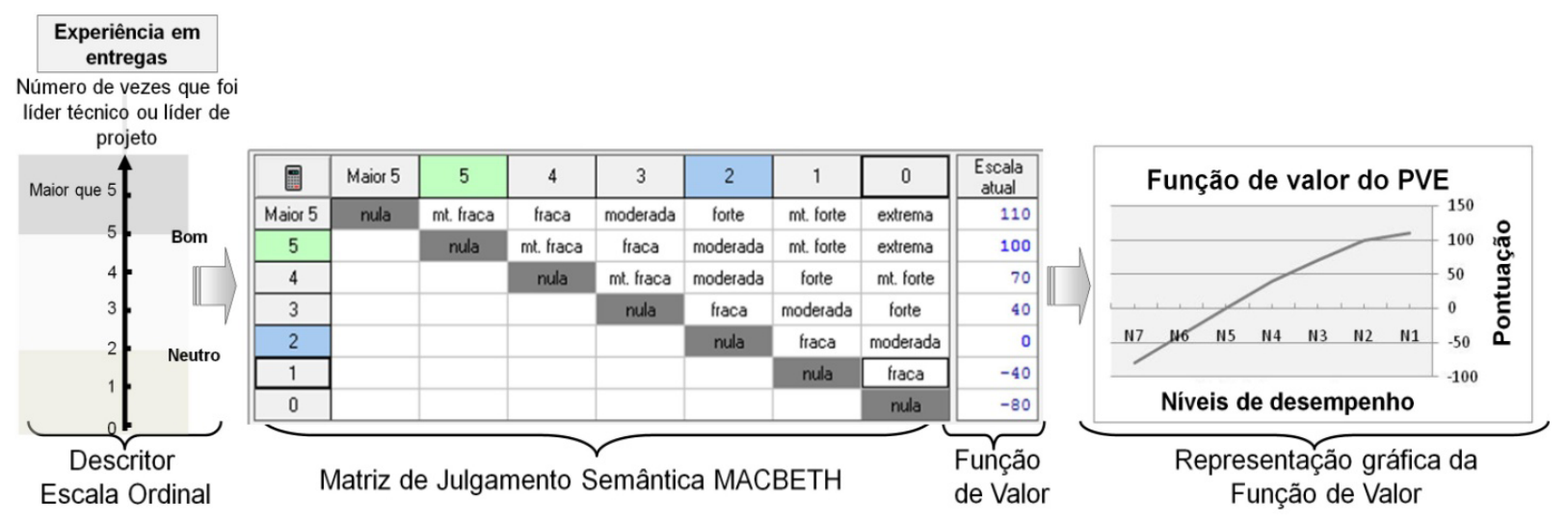

Figura 3 - Transformação do Descritor "Experiência em entregas” em Função de Valor por meio do Método MacBeth

Fonte: O autor (2012)

Após completar a etapa de funções de valor, é permitido ao decisor avaliar o impacto local das ações no nível operacional (local), realizando a mensuração cardinal. Esse processo expande o conhecimento e abre um leque de oportunidades de ações para o decisor, mas ainda restrito ao nível operacional e não aos níveis táticos e estratégicos, através das comparações e níveis de impacto entre escalas (descritores). Para essa demanda, faz-se necessário ainda construir as taxas de compensações e essa etapa será apresentada a seguir.

\subsection{Taxas de Compensação ou Substituição}

As taxas de compensação (vulgarmente conhecidas como pesos) são constantes de escala que permitem expandir a análise local (único descritor) para valores globais (todos descritores). Dessa forma, atribui-se uma taxa de compensação para cada descritor que define a importância que cada descritor tem em relação aos demais (ENSSLIN, 2001).

Para obtenção das Taxas de Compensação ou Substituição foi adotado o método de Comparação Par-a-Par contemplado pelo Macbeth. Considerando o processo através do método Macbeth, são realizadas três etapas: Identificação das alternativas, ordenação das alternativas e construção da matriz de julgamento semântica.

Para exemplificar o processo, é demonstrada a obtenção das taxas para os PVEs: Ferramentas Finais, Testes Finais e Decisão do Negócio. De acordo com Ensslin et al. (2010), foram estabelecidas quatro alternativas: N1- Ferramentas Finais, N2- Testes Finais, N3- Decisão do Negócio e N4- Neutro. A alternativa N1 apresenta o PVE-Ferramentas Finais no nível Bom e as demais alternativas no nível Neutro, sendo que o mesmo foi aplicado para as alternativas N2 e N3. No caso da alternativa N4, todos os PVEs permeneceram no nível Neutro. Já a ordenação foi realizada a partir da Matriz de Roberts.

Por fim, utilizou-se o mesmo processo acima apresentado para a transformação da escala ordinal em Função de Valor para as Taxas de Compensação com o uso do programa M-Macbeth conforme Figura 4. Nesse caso, a alternativa $\mathrm{N} 2=45>\mathrm{N} 3=35>\mathrm{N} 1=20>\mathrm{N} 4$ (tudo inferior $)=0$.

\begin{tabular}{|c|c|c|c|c|c|}
\hline 畻 & [N2 ] & [N3 ] & [N1] & [ tudo inf. ] & $\begin{array}{c}\text { Escala } \\
\text { atual }\end{array}$ \\
\hline [N2 ] & nula & mt. fraca & mt. forte & extrema & 45 \\
\hline [N3] & & nula & forte & mt. forte & 35 \\
\hline [N1] & & & nula & forte & 20 \\
\hline [ tudo inf.] ] & & & & nula & 0 \\
\hline
\end{tabular}

Figura 4 -Taxas de substituição calculadas no M-Macbeth para os PVEs Ferramentas Finais, Testes Finais e Decisão do Negócio 
O processo supracitado foi realizado para todo o Modelo, transportando as taxas de substituição criadas para a Estrutura Hierárquica de Valor conforme apresentado na Figura 5 para o PVF9 - Avaliação Final, permitindose a análise global das alternativas analisadas no nível local e estratégico. De posse desse modelo, o Líder de Tecnologia (decisor) terá uma ferramenta de apoio a decisão durante o processo de alocação de pessoas com base nas suas competências, sendo evidenciadas potencialidades e oportunidades de aperfeiçoamento.

\section{GESTÃO POR COMPETÊNCIA DOS PROFISSIONAIS ATRAVÉS DE UM MODELO MULTICRITÉRIO CONSTRUTIVISTA}

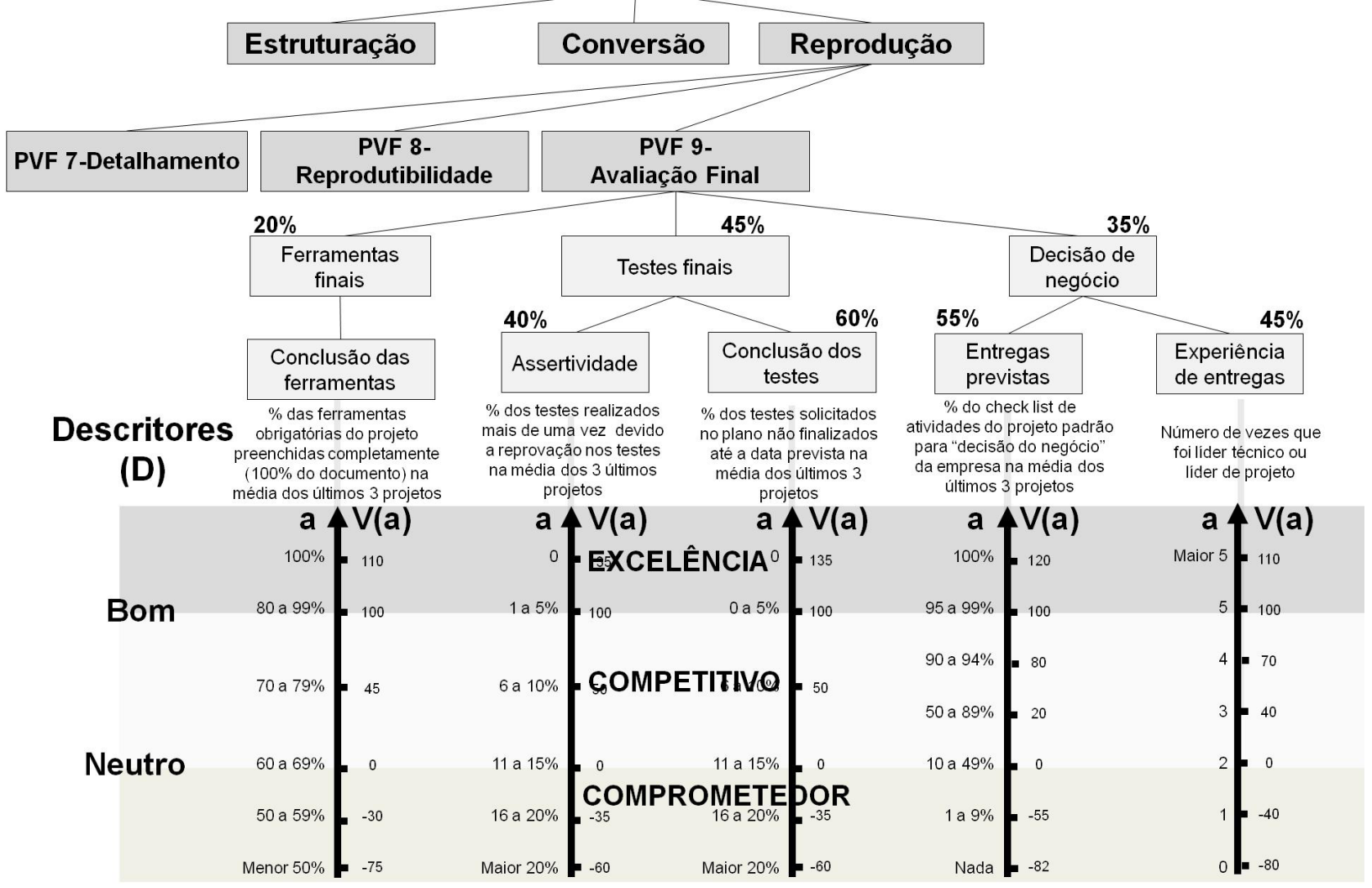

Figura 5 - Estrutura Hierárquica de Valor para o PVF 9-Avaliação Final e Taxas de Substituição

Fonte: O autor (2012)

\subsection{Identificação do Perfil de Impacto das Alternativas}

Com o modelo construído após completar as etapas anteriores do método MCDA-C, é possível ao gestor mensurar as alternativas que julgar relevantes e analisar o perfil de impacto delas no nível local (descritor) e estratégico (resultado global). Dessa forma e com todo o conhecimento adquirido pelo processo, é estabelecido o status quo.

O detalhamento científico com relação à equação para obtenção do valor global ao considerar uma ação "a”, pode ser verificado abaixo na equação 1 :

$$
V_{P V F k}(a)=\sum_{i=1}^{n k} w_{i, k} \cdot V_{i, k}(a)
$$

Com:

$$
\begin{aligned}
& V_{P V F k}(a): \text { valor global da ação } a \text { do } \mathrm{PVF}_{\mathrm{k}} ; \\
& V_{i, k}(a) \text { : valor parcial da ação } a \text { no critério } \mathrm{i}, \mathrm{i}=1, \ldots \mathrm{n} ; \\
& a: \text { nível de impacto da ação } a ; \\
& w_{i, k}: \text { taxas de substituição do critério } \mathrm{i}, \mathrm{i}=1, \ldots \mathrm{n} ; \\
& \mathrm{n}_{\mathrm{k}}: \text { número de critérios do } \mathrm{PVF}_{\mathrm{k}} ; \\
& \mathrm{k}: \text { número do PVF; }
\end{aligned}
$$

Para realizar a análise completa de uma ou mais alternativas no modelo, parte-se da equação geral (1) e somamse os nove PVFs construídos conforme Equação (2): 


$$
\begin{aligned}
& V_{G l o b a l}(a)=w_{1} \cdot V_{P V F_{1}}(a)+w_{2} \cdot V_{P V F_{2}}(a)+w_{3} \cdot V_{P V F_{3}}(a)+w_{4} \cdot V_{P V F_{4}}(a)+ \\
& +w_{5} \cdot V_{P V F_{5}}(a)+w_{6} \cdot V_{P V F_{6}}(a)+w_{7} \cdot V_{P V F_{7}}(a)+w_{8} \cdot V_{P V F_{8}}(a)+w_{9} \cdot V_{P V F_{9}}(a)
\end{aligned}
$$

Com:

$\mathrm{V}_{\text {Global }}(\mathrm{a})=$ Pontuação global para o modelo construído;

$$
\begin{aligned}
& \mathrm{V}_{\mathrm{PVF}}(a)=0,20\left(1 . \boldsymbol{V}_{\text {Conclusão das Ferramentas }}\right)+0,45\left(0,4 . \boldsymbol{V}_{\text {Assertividade }}+0,6 \cdot \boldsymbol{V}_{\text {Conclusão dos Testes }}\right)+0,35\left(0,55 . \boldsymbol{V}_{\text {Entregas }}\right. \\
& + \text { 0,45. } \boldsymbol{V}_{\text {Experiência de Entregas) }}
\end{aligned}
$$

A equação é completada utilizando todos os Pontos de Vistas, do nível mais inferior aos superiores, de forma somatória e multiplicando as funções pelas taxas de compensação na Estrutura Hierárquica de Valor.

A utilização da alternativa referencial (status quo) permite ao Líder de Tecnologia fazer o diagnóstico do problema analisado e assim construir conhecimento acerca da performance crítica (nível comprometedor), os aspectos que necessitam ser fortalecidos (nível competitivo) e também aqueles que podem ser considerados benchmark (excelência).

No presente estudo de caso, o Líder de Tecnologia tomou a decisão de avaliar duas opções de Engenheiros no modelo para verificar o impacto no resultado de acordo com o grau de habilidade e competência de ambos. A alternativa 1 que também foi a considerada (Status Quo) tratava-se um Engenheiro Junior com 5 anos de experiência, com muitas habilidades exigidas pela função e com resultados considerados na média pelos gestores. Já no caso da alternativa 2, o Líder
Para detalhar o processo e apresentar os valores adotados no modelo, será apresentado na equação (3) o PVF9Avaliação Final. Utilizou-se como alternativa base (status quo) um Engenheiro com 5 anos de experiência.

de Tecnologia resolveu aproveitar o modelo para ser arrojado na possível utilização de um Engenheiro recém contratado, com o objetivo de ter o conhecimento de um resultado comprometedor (considerando o cargo e não sua competência) ou no caso de um resultado favorável, verificar a possibilidade de utilizá-lo para atender as demandas do projeto.

Para visualizar gráfica e numericamente esse estudo, é apresentada na Figura 6 a análise de impacto das duas alternativas com foco no PVF9. É notório tanto pelo gráfico quanto pelo resultado numérico que a alternativa 2 fica distante da alternativa 1. O Engenheiro Junior (Alternativa 1) alcançou resultado competitivo (valor global 32,65) já o Engenheiro recém contratado (Alternativa 2) foi plotado na performance comprometedora (resultado global -35,10). Segundo o julgamento de valores do decisor (nível esperado de desempenho), a própria alocação do Engenheiro Junior nesse projeto já seria equivocada, uma vez que seria esperado um resultado abaixo da média e no caso o Engenheiro recém contratado, uma escolha que colocaria em risco o projeto. 

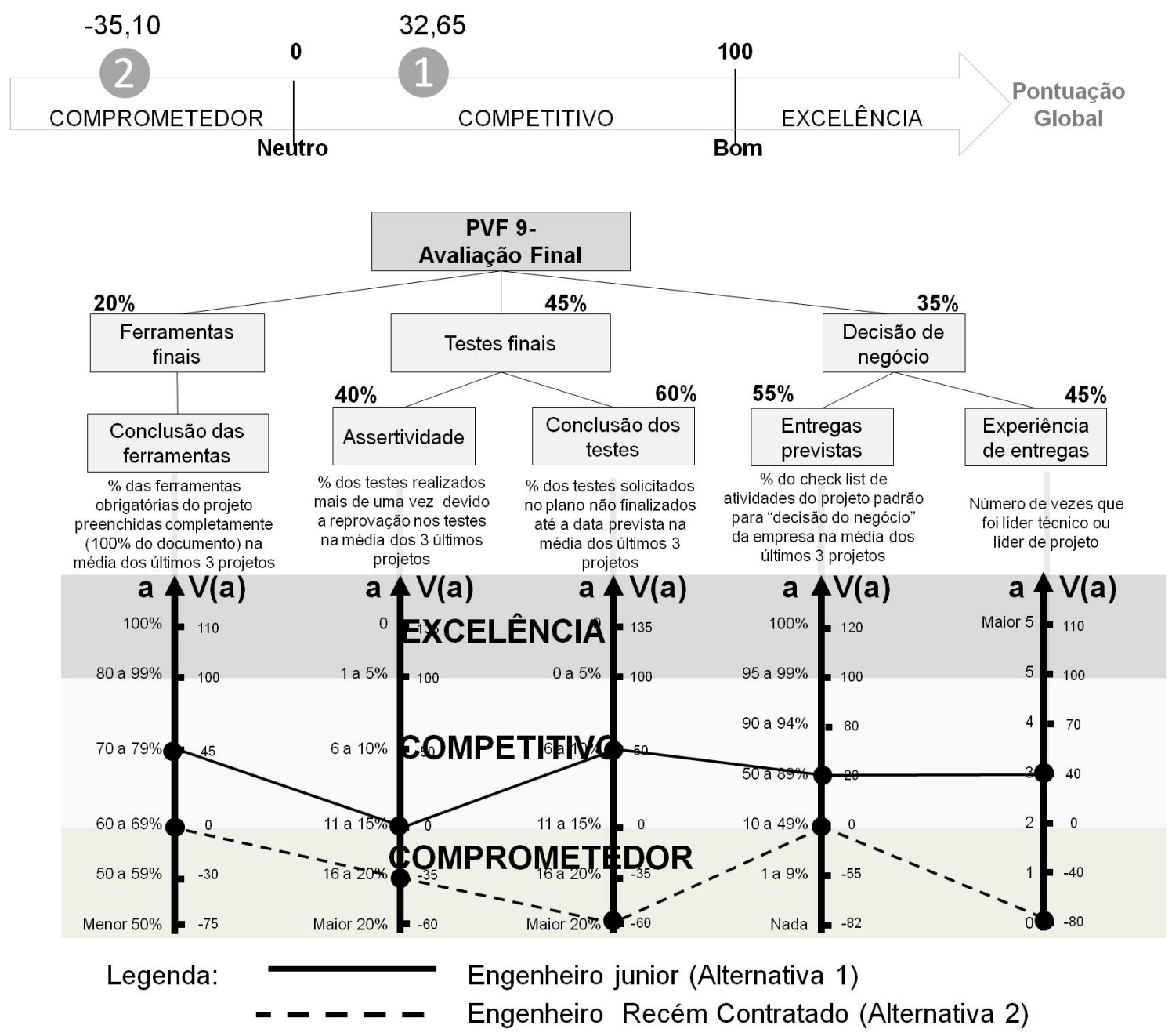

Figura 6- Perfil do Status do Engenheiro Junior x Recém contratado no PVF 9 - Avaliação Final

Fonte: O autor (2012)

O processo supracitado foi realizados para todas as opções de Engenheiros disponíveis ao Líder de Tecnologia para alocação no projeto específico e a opção anteriormente citada, Alternativa 1, foi a que obteve o melhor resultado com pontuação de 32,65.

Assim, novamente de posse dessas análises permitidas através do modelo de apoio a decisão, o decisor verificou que dentre as opções disponíveis, a melhor opção ainda precisava de aperfeiçoamento para alcançar um resultado esperado (Nível Bom ou superior). Esse processo para aperfeiçoamento será apresentado na Fase de Recomendações.

\subsection{Análise de Sensibilidade}

O método MCDA-C recomenda a realização da etapa de Análise de Sensibilidade antes de seguir para a fase de Recomendações para garantir a robustez do modelo construído. Com esse objetivo, o processo de Análise de
Sensibilidade foi realizado segundo Ensslin et al. (2001) nas taxas de todos os PVEs do modelo para Gestão por competência dos profissionais.

\section{Fase de Recomendações}

A fase de Recomendações é o momento em que o decisor pode aproveitar todo o conhecimento construído e materializado através do modelo. $\mathrm{O}$ modelo tem alto potencial por gerar oportunidades de melhoria, pois nele já estão detalhados os níveis de performance das alternativas analisadas e o gestor já sabe como melhorar ou piorar, além do impacto de cada decisão.

\subsection{Ações de Aperfeiçoamento}

Retornando-se a alternativa 1 (Engenheiro Junior) que obtinha a pontuação de 32,65 , constatado que estava abaixo do esperado em termos de desempenho segundo o julgamento de valores do decisor e necessitava de 
aperfeiçoamento para alcançar uma performance em nível Bom ou superior para o projeto em estudo, buscouse implementar um plano de ação de aperfeiçoamento.

Tendo em vista o curto prazo de tempo entre a execução do plano e o início do projeto, disponibilidade de pessoas para realizar os treinamentos e as demandas de projetos já em execução, o decisor solicitou ao facilitador que fizesse a análise do impacto no resultado global ao considerar a elevação em um nível para cada critério.
Com o procedimento ilustrado para o PVF9 - Análise Final com seus 5 Descritores (Conclusão das Ferramentas; Assertividade; Conclusão dos Testes; Entregas Previstas; e Experiência de Entregas) e considerando a elevação em um nível para cada um dele com as ações de melhorias ( $\beta 1 ; \beta 2 ; \beta 3 ; \beta 4$; e $\beta 5)$, obtiveram-se seus respectivos impactos globais no PVF9 e consequentemente um resultado total de 49,78 conforme indicado na Figura 7.

\begin{tabular}{|c|c|c|c|c|c|c|}
\hline \multicolumn{7}{|c|}{ PLANO DE AÇÃO } \\
\hline Ação & PVE & Descritor & Nivel Atual & Nivel Meta & (D) & $\begin{array}{l}\text { Impacto Global } \\
\text { no PVF9 }\end{array}$ \\
\hline$\beta 1$ & $\begin{array}{l}\text { Conchusão das } \\
\text { ferramentas }\end{array}$ & $\begin{array}{l}\% \text { das ferramentas obrigatórias do projeto preenchidas } \\
\text { completamente }(100 \% \text { do documentb) na média dos } \\
\text { ültimos } 3 \text { projetos }\end{array}$ & 70 a $79 \%$ & 80 a $99 \%$ & 72 & 11 \\
\hline$\beta 2$ & Assertividade & $\begin{array}{l}\% \text { dos testes realizados mais de uma vez devido a } \\
\text { reprovacão nos testes na média dos } 3 \text { últimos projetos }\end{array}$ & 11 a $15 \%$ & 6 a $10 \%$ & 73 & 9 \\
\hline$\beta 3$ & $\begin{array}{c}\text { Conchusão dos } \\
\text { estes }\end{array}$ & $\begin{array}{l}\% \text { dos testes solicitados no plano não finalizados até a data } \\
\text { prevista na média dos últimos } 3 \text { projetos }\end{array}$ & 6 a $10 \%$ & 0 a $5 \%$ & 74 & 13,5 \\
\hline$\beta 4$ & Entregas previstas & $\begin{array}{l}\% \text { do check list de atividades do projeto padrão para } \\
\text { "decisão do negócio" da empresa na média dos últimos } 3 \\
\text { projetos }\end{array}$ & 50 a $89 \%$ & 90 a $94 \%$ & 75 & 11,55 \\
\hline$\beta 5$ & $\begin{array}{c}\text { Experiência de } \\
\text { entregas }\end{array}$ & Número de vezss que foi lider técrico ou lider de projeto & 3 & 4 & 76 & 4,73 \\
\hline & & & & Inpacto & Cotal & 49,78 \\
\hline
\end{tabular}

Figura 7 - Plano de ação de aperfeiçoamento do Engenheiro Junior com foco no PVF9

Fonte: O autor (2011)

O plano de ação foi legitimado pelo decisor e executado após dois meses de trabalho. Com a realização integral de todas as ações de melhoria, foi possível alcançar a pontuação 82,43 no PVF9, partindo-se do valor base 32,65 e obtendo um incremento de 49,78.

Vale destacar que as ações de aperfeiçoamento foram todas atividades internas e que não dependiam de recursos financeiros e sim, o entendimento das deficiências, priorização e conhecimento do problema enfrentado.

\section{Considerações Finais}

A pergunta de pesquisa "Como realizar a alocação de pessoas para atender as demandas de projetos para o contexto avaliado com visão sistêmica e alavancar vantagem competitiva para organização?", foi respondida com o cumprimento do objetivo do estudo "realização da gestão por competência dos profissionais através de um modelo multicritério construtivista para atender as demandas de conhecimento dos projetos e criar assim um diferencial competitivo".

Para esse contexto, foi adotado o método MCDA-C como instrumento de intervenção tendo em vista à complexidade no processo de identificação dos objetivos e gestão do conflito de interesses dos atores envolvidos no problema e por fim, o interesse na avaliação do impacto de cada decisão no resultado global.

No sentido de detalhar os meios para o atendimento ao objetivo geral, apresentam-se as etapas do processo adotado pelo MCDA-C em suas três fases Estruturação, Avaliação e Recomendações, conforme Figura 1. Com relação à contextualização do problema, foi realizada na fase Estruturação, mais especificamente com abordagem soft para estruturação, tendo definido o estudo de caso numa empresa Multinacional que desenvolve e fabrica eletrodomésticos. Além disso, o sistema de atores foi definido e apresentado no Quadro 1.

Para a identificação dos critérios/KPIs segundo o juízo de valores do decisor no contexto analisado, foram realizadas reuniões formais com o decisor e outras conversas informais com os atores envolvidos com o objetivo de extrair do Líder de Tecnologia toda informação necessária para alimentar o processo e posteriormente construir o modelo. Com relação aos critérios, na etapa de Família de Pontos de Vista, foram identificados 80 Elementos Primários de Avaliação (EPAs) que foram transformados 


\section{Produto \& Produção, vol. 13, n. 3, p. 105-120, out. 2012}

em Conceitos (vide Quadro 2) que representaram os candidatos a critérios para o modelo.

Com posse dos conceitos e para atender a criação das escalas na forma ordinal, apresenta-se principalmente a etapa de Construção dos Descritores com a execução dos Mapas Cognitivos conforme Figura 2, Árvore de Pontos de Vistas Fundamentais e Estrutura Hierárquica de Valores. Seguindo-se o processo supracitado, foram identificados 76 critérios/KPIs que representam os aspectos julgados como relevantes e necessários para o decisor no contexto. No caso da transformação da escala ordinal em cardinal, destacam-se as etapas de Análise de Independência e principalmente a etapa de Construção das Funções de Valor, na qual se destaca a utilização do método Macbeth, tendo em consideração a vasta aplicação dos pesquisadores no mundo científico e fundamentação teórica, e também o uso programa M-MacBeth, que permitiu a criação das escalas cardinais com base em modelos de programação linear. O resultado final para um descritor "Experiência em Entregas" pode ser conferido na Figura 3.

Com relação à mensuração com diagnóstico numérico e gráfico da situação atual (status quo) tanto em nível local quanto global, foi possível atender primeiramente com a identificação das taxas de Compensação conforme apresentado na Figura 4, um exemplo para os PVEs Ferramentas Finais, Testes Finais e Decisão do Negócio o que resultou nas taxas $20 \%, 45 \%$ e $35 \%$, sendo que o mesmo processo foi realizado para todos os PVEs e as taxas transportadas para o modelo (vide Figura 5, a ilustração para o PVF9-Avaliação). No segundo momento para o diagnóstico numérico e gráfico, realizou-se a etapa de Identificação do perfil de impacto das alternativas, na qual a equação 2 ilustrou numericamente o impacto global para uma ação "a" e a equação 3, a pontuação global para uma alternativa escolhida (Engenheiro Junior) com foco no PVF9Avaliação Final, obtendo o impacto global de 32,65 e servindo como Status Quo. Já a representação gráfica pode ser apresentada principalmente pela Figura 6 com Perfil do Status do Engenheiro Junior x Recém contratado no PVF 9.

Por fim, tendo em vista a realização das ações de aperfeiçoamento com pleno entendimento do impacto de cada decisão tomada, é citada a fase de Recomendações. Com o procedimento ilustrado para o PVF9 - Análise Final com seus 5 Descritores (Conclusão das Ferramentas; Assertividade; Conclusão dos Testes; Entregas Previstas; e Experiência de Entregas), foi estabelecido um plano de ação apresentado na Figura 7 que possibilitou um incremento no resultado global de 49,78. Assim, com a realização integral de todas as ações de melhoria, foi possível alcançar a pontuação final de 82,43 no PVF9, partindo-se do valor base 32,65 e com um aumento de 49,78.
O Modelo de Avaliação de Desempenho construído serviu como ferramenta de apoio ao Gestor durante a alocação de recursos tendo em consideração o grau de conhecimento, habilidade e aptidão das pessoas quando do desenvolvimento de seus projetos.

Apresenta como limitação de pesquisa, o método MCDA-C permitir aplicações do modelo construído para o contexto para o qual foi concebido e os atores envolvidos, assim restringir as aplicações genéricas. Além disso, demanda alto grau de envolvimento por parte do Líder de Tecnologia (decisor) ao longo do processo e esses líderes normalmente dispõem de pouquíssimo tempo disponível.

É recomendado para as pesquisas futuras, realizar a avaliação do mesmo problema, porém com um decisor diferente de forma à verificar o impacto no resultado final e assim, compreender as vulnerabilidades de tal decisão ao se comparar com o modelo inicial proposto para o Líder de Tecnologia.

\section{Referências}

AZEVEDO, J. Aplicação da metodologia multicritério de apoio à decisão na seleção de centros de usinagem para uma central de usinagem. Dissertação (Mestrado em Engenharia de Produção) - Universidade Federal de Santa Catarina, Florianópolis, 2001.

BAIRD, L.; MESHOULAM, I. Managing two fits of strategic human resource management. Academy of Management Review, p. 116-128, 1988.

BANA E COSTA, C. A. Três convicções fundamentais na prática do apoio à decisão. Pesquisa Operacional, v.13, n.1, p. 9-20. 1993.

BANA E COSTA, C. A.; ENSSLIN, L. Decision support systems in action: integrated application in a multicriteria decision aid process. European Journal of Operational Research, v. 113, n. 2, p. 315-335, 1999.

BANA E COSTA, C. A. B.; DE CORTE, J. M.; VANSNICK, J. C. On the mathematical foundations of macbeth. In: Multicriteria Decision Analysis: state of the art survey [edited by Greco, J. F. \& Ehrgott, S. M.], Springer Verlag, Boston, Dordrecht, London, p. 15-24, 2005.

BARZILAI, J. On the foundations of measurement: IEEE, p. 401-406, v. 1. 2001.

BEARDWELL, J.; CLAYDON, T. Human resource management: a contemporary approach: Pearson Education. 2007. 
BRESTER, C.; TREGASKIS, O.; HEGEWISCH, A.; MAYNE, L. Comparative research in human resource management: a review and an example. International Journal of Human Resource Management, v. 7, n. 3, p. 585-604, 1996.

BUCK, J. M.; WATSON, J. L. Retaining staff employees: The relationship between human resources management strategies and organizational commitment. Innovative Higher Education, v. 26, n. 3, p. 175-193, 2002.

CAI, J.; LIU, X.; XIAO, Z.; LIU, J. Improving supply chain performance management: A systematic approach to analyzing iterative KPI accomplishment. Decision Support Systems, v. 46, p. 512-521, 2009.

CHAN, F. T. S. Performance measurement in a supply chain. International Journal of Advanced Manufacturing Technology, v. 21, p. 534-548, 2003.

EDEN, C.; ACKERMANN, F.; CROPPER, S. The analysis of cause maps. Journal of Management Studies, v. 29, n. 3, p. 309-324, 1992.

ENSSLIN, L.; DUTRA, A.; ENSSLIN, S. R. MCDA: a constructivist approach to the management of human resources at a governmental agency. International Transactions in Operational Research, v. 7, n. 1, p. 79-100. 2000.

ENSSLIN, L., GIFFHORN, E., ENSSLIN, S. R., PETRI, S. M.; VIANNA, W. B. Avaliação do desempenho de empresas terceirizadas com o uso da metodologia multicritério de apoio à decisão-construtivista. Pesquisa Operacional, v. 30, n. 1, p. 125-152. 2010.

ENSSLIN, L.; MONTIBELLER, G. N.; NORONHA, S. M. Apoio à decisão: metodologias para estruturação de problemas e avaliação multicritério de alternativas. Florianópolis: Insular, 2001.

ENSSLIN, L.; VIANNA, W. B. O design na pesquisa quali-quantitativa em engenharia de produção-questões epistemológicas. Revista Produção Online, v. 8, n. 1, 2008.

GANN, D. M.; SALTER, A. J. Innovation in projectbased, service-enhanced firms: the construction of complex products and systems. Research Policy, v. 29, n. 7-8, p. $955-972,2000$.

GIL, A. C. Métodos e técnicas de pesquisa social. 5. ed. São Paulo: Atlas. 1999.

GOODWIN, P.; WRIGHT, G. Decision Analysis for Management Judgment: John Wiley \& Sons, Chichester, 1998.
HENDRIKS, M.; VOETEN, B.; KROEP, L. Human resource allocation in a multi-project $R \& D$ environment: Resource capacity allocation and project portfolio planning in practice. International Journal of Project Management, v. 17, n. 3, p. 181-188, 1999.

HUSELID, M. A. The impact of human resource management practices on turnover, productivity, and corporate financial performance. Academy of Management Journal, p. 635-672, 1995.

IGARASHI, D. C. C.; ENSSLIN, S. R.; ENSSLIN, L.; PALADINI, E. P. A qualidade do ensino sob o viés da avaliação de um programa de pós-graduação em contabilidade: proposta de estruturação de um modelo híbrido. Revista de Gestão USP, v. 43, p. 117-137, 2008.

JACKSON, S. E.; SCHULER, R. S. Understanding human resource management in the context of organizations and their environments. Strategic human resource management, p. 4-28, 1999.

JIMENEZ, J. B.; LORENTE, J. J. C. Environmental performance as an operations objective. International Journal of Operations \& Production Management, v. 21, n. 12, p. 1553-1572, 2001.

KEENEY, R. L. Value-focused thinking: A path to creative decision making. Harvard University Press. 1992

LADO, A. A.; WILSON, M. C. Human resource systems and sustained competitive advantage: A competency-based perspective. Academy of Management Review, p. 699-727, 1994.

LANDRY, M. A note on the concept of'problem'. Organization Studies, v. 16, n. 2, p. 315. 1995.

RABECHINI, R.; CARVALHO, M. M.; LAURINDO, F. J. B. Fatores críticos para implementação de gerenciamento por projetos: o caso de uma organização de pesquisa. Revista Produção, v. 12, n. 2, p. 28-41, 2002.

ROY, B. Decision science or decision-aid science? European Journal of Operational Research, v. 66, n. 2, p. 184-203, 1993.

ROY, B. On operational research and decision aid. European Journal of Operational Research, v. 73, p. 23-26, 1994.

ROY, B. Multicriteria Methodology for Decision Aiding. Kluwer Academic Publishers, Dordrecht, 1996.

ROY, B. Paradigms and challenges. In: Multiple Criteria Decision Analysis: state of the art surveys. New York: Springer, p. 03-24, 2005. 
120 Produto \& Produção, vol. 13, n. 3, p. 105-120, out. 2012

ROY, B.; BOUYSSOU, D. Decision-aid: an elementary introduction with emphasis on multiple criteria. Information Science and Technology, v. 2, p. 109-123, 1993.

SHENHAR, A. J. One size does not fit all projects: exploring classical contingency domains. Management Science, p. 394-414, 2001.

SKINNER, W. The productivity paradox. Management Review, v. 75, n. 9, p. 41-45, 1986.

WRIGHT, P. M.; DUNFORD, B. B.; SNELL, S. A. Human resources and the resource based view of the firm. Journal of Management, v. 27, n. 6, p. 701, 2001.

ZIMMERMANN, H. J. An application-oriented view of modeling uncertainty. European Journal of Operational Research, v. 122, n. 2, p. 190-198, 2000.

Recebido em 21/02/2012. Aceito em 31/07/2012. 